\title{
Influence of Geological Situation on the Behavior of Twin Tunnel
}

\author{
Seok-Won Lee', Hoon Jung ${ }^{2}$, Sung-Yun Kim² \\ ${ }^{1}$ Konkuk University \\ Songpa-Gu, Seoul, Korea \\ swlee@konkuk.ac.kr \\ ${ }^{2}$ Konkuk University \\ Gwanggin-Gu, Seoul, Korea \\ cu_verine@naver.com; chupchupe@naver.com
}

\section{Extended Abstract}

According to the standard specification of tunnel construction, it is reported that, in order not to give any influence on each other, the distance between the tunnel centers of twin tunnel should be greater than the twice of the excavation width when they are constructed in the elastic soils, and should be greater than 5 times in case of the soft ground. Generally, the distance between the tunnel centers is desired as 2.5 times of the excavation width. But it is just a recommendation not from the engineering analysis but from the design experience.

In this study, to find out the mutual interference influence according to the separated distance of adjacent tunnels, the particle flow code(PFC) model of the distinct element method was used, and the interactive behavior of tunnels with the change of separation distance and coefficient of lateral earth pressure. This was to complement the limit of previous analysis methods based on the continuum mechanics. And, for the verification of numerical analysis model, the results were compared with those of the scaled model test under the same condition.

For the scaled chamber test, a soil tank was produced. As the material for the model, the mixture of sands, plasters, and water was used. The dimension of model was $1000 * 600 * 100 \mathrm{~mm}$ (length*height*width) and the tunnel was simulated as a circle with the diameter of $140 \mathrm{~mm}$. The scaled model experiment was carried out for 8 cases while changing the separation distances between adjacent tunnel and the coefficient of lateral earth pressure.

The conclusions of them are as follows:

(1) The displacement around the tunnel according to the separation distance between the tunnel centers tends to be greater in all measuring points as the distance becomes shorter. The displacement of pillar part between the tunnels exactly changes in proportion with the separation distance until the pillar is failed under the duplicated loads. But, in case that the separation is greater than $2.0 \mathrm{D}$, the interference effect diminishes a lot, as noted in other previous studies.

(2) The displacement around the adjacent tunnels according to the change of coefficient of earth pressure decreases as the coefficient of earth pressure increases under the same separation distance.

(3) As a result of verification of numerical analysis through the scaled model test, the pattern and the amount of displacement were shown almost same. The cracking pattern was a little different around the model boundary because of the difference in physical test condition between the scaled model test and the numerical analysis model, but nearly same around the tunnel. From this result, it could be confirmed that PFC is an appropriate numerical analysis method in simulating the behavior of adjacent tunnels.

\section{References}

[1] D. D. Tannant and C. Wang, "Thin tunnel liners modelled with particle flow code," Engineering Computations, vol. 21, no. 2/3/4, pp. 318-342, 2003.

[2] B. Yang, Y. Jiao and S. Lei, "A study on the effects of microparameters on macroproperties for specimens created by bonded particles," Engineering Computations: International Journal for Computer-Aided Engineering and Software, vol. 23, no. 6, pp. 607-631, 2006.

[3] B. L. Chu, S. C. Hsu, Y. L. Chang and Y. S. Lin, "Mechanical behavior of a twin-tunnel in multi-layered formations," Tunnelling and underground space technology, vol. 22, no. 3, pp. 351-362, 2007. 